\title{
VISUALIZACIÓN DEL ACOSO EN TENDEDEROS UNIVERSITARIOS; UNA CONSTRUCCIÓN DE PAISAJES LINGÜÍSTICOS
}

\author{
VISUALIZATION OF HARASSMENT IN UNIVERSITY TENDEDEROS; A \\ CONSTRUCTION OF LINGUISTIC LANDSCAPES \\ (iD) Cándido Chan Pech ${ }^{*}$ \\ c.chan@live.com.mx \\ ${ }^{1}$ Universidad Autónoma de Chiapas, México \\ *Correspondencia: Cándido Chan Pech. Email: c.chan@live.com.mx
}

Recibido: 05.08.21 | Aprobado: 08.09.21

\section{RESUMEN}

Los espacios universitarios son escenarios donde la violencia de género y acoso sexual vive en la cotidianidad, pocas veces han sido exhibidas por la dominación del poder del sistema patriarcal además de la amenaza de coacción en consecuencia. Sin embargo, la necesidad colectiva de visualizarla en los llamados tendederos, donde las alumnas escriben en hojas de papel su denuncia de manera anónima; son prácticas que están visualizando y exhibiendo las representaciones del acoso sexual: miradas y proposiciones. Desde un tratamiento semiótico-lingüístico se atendió la invitación para dilucidar el sentido de tal práctica como una puesta colectiva de visualizar lo inaparente de la violencia en la universidad. La intención principal de este artículo es plantear el tendedero como la construcción de un paisaje lingüístico y como práctica de visualización del acoso sexual como referente inmediato de las conceptualizaciones en los espacios de la universidad.

Palabras clave: Violencia de género, acoso sexual, espacios universitarios, paisajes lingüísticos.

\begin{abstract}
University spaces are scenarios where gender violence and sexual harassment live in everyday life, they have rarely been exhibited by the domination of the power of the patriarchal system in addition to the threat of coercion accordingly. However, the collective need to visualize it in the so-called clotheslines, where the students writeon sheets of paper their anonymous complaint; are practices that are visualizing and exhibiting the representations of sexual harassment: looksand propositions. From a semiotic-linguistic treatment, the invitation to elucidate the meaning of such a practice as a collective setting of visualizing the inappparent of violence in the university was heeded. The main intention of this article is to propose the clothesline as the construction of a linguistic landscape and as a practice of visualization of sexual harassment as an immediate reference of the conceptualizations in the spaces of the university.
\end{abstract}

Keywords: Gender violence, sexual harassment, university spaces, linguistic landscapes. 


\section{INTRODUCCIÓN}

La necesidad de la visualización y denuncia del acoso sexual como violencia de genero se ha afianzado en las tendencias globales, nacionales y locales. Inicialmente el movimiento metoo de Tarana Burke sirvió como parteaguas, alcanzando un impacto en los acontecimientos del 2017, específicamente con las acusaciones de acoso sexual (en contra de un productor cinematográfico de Hollywood) a actrices, modelos y productores. Por otro lado, el eufemismo provocado por Alyssa Milano quien viralizó el hashtag \#Metoo con casi 14 millones de tweets invitando a denunciar el acoso sexual. Aunado a ello, está el caso del protagonista de una conocida serie de Netflix, acusado de hostigamiento sexual por muchos años, que dio lugar a múltiples acusaciones a comediantes, productores musicales y varios actores. En tan solo un año, en Estados Unidos fueron, cientos de personajes fueron señalados de hostigamiento sexual y abuso de poder, extendiéndose a diversos ámbitos como la política, artistas, deportes, finanzas, periodistas y desarrolladores de tecnología (Muñoz-Saavedra, et.al. 2019)

En México, actrices expresaron el acoso de manera sistemática al exponer que Televisa invitaba a eventos con publicistas y las promocionaba sexualmente en un catálogo por grandes sumas de dinero. En 2017, comediantes mexicanas como integrantes del gremio específico, firmaron una declaración con el hashtag \#Yaestuvo en donde exigieron un alto al hostigamiento y abuso sexual. En 2018, varias actrices y deportistas fueron entrevistadas por Carmen Aristegui, en calidad de víctimas de acoso sexual y psicológico en sus espacios de trabajo como consecuencias de situaciones de poder.

En marzo de 2019, Ana G. González, publicó en Twitter que un escritor mexicano golpeó, sedujo, embarazó y amenazó a más de una decena de chicas. Otras evidenciaron lo mismo: promoviendo la creación de \#Metooescritoresmexicanos y @metooescritores para denunciar a supuestos acosadores; 134 escritores fueron indicados, ocho, por más de cinco personas. Además, fueron admitidas 120 denuncias en contra 119 hombres reporteros, fotógrafos, editores y columnistas de medios en México. El Twitter ha sido utilizado constantemente para exhibir el hostigamiento sexual en industrias como el periodismo, el cine, el teatro, la política, la academia, la publicidad y la programación.

En México, las denuncias en redes sociales contra acosadores tomaron fuerza; en el 2019 el movimiento trascendió al grado de que las mujeres se manifestaron continuamente en las calles y en las redes sociales; no solo famosas sino de todos ámbitos atreviéndose a denunciar su caso. Se inició la llamada revuelta tuitera con los hashtags metoo, evidenciando así que en todos los gremios hay mujeres, la mayoría desde el anonimato, dispuestas a contar su historia. En protesta, en marzo del año pasado, las mujeres salieron a las calles, exhibiendo casos extremos y concretos de feminicidio utilizado el performance; "El violador eres tú", "Nos están matando" entre otros. A raíz de dos feminicidios: Ingrid y Fátima, se promovió el movimiento \#UnDíaSinNosotras, lanzados por colectivos feministas, a un paro nacional de mujeres para el 9 de marzo de este año cuyo objetivo fue el de protestar contra los feminicidios, la violencia de género y el acoso sexual. Estas tendencias, han sido señaladas por Cynthia Dackow (2020) como "puntos básicos de confluencia que les permiten enfrentar al poder patriarcal con cohesión y coherencia discursiva", conformando así un "paisaje semiótico disidente" que "intenta deconstruir las representaciones sociales cristalizadas y naturalizadas" (p. 3). 
Estas tendencias repercutieron en las universidades con la denuncia de violencia física, sexual y psicológica. Las alumnas se adueñaron de espacios para visibilizar la violencia y el acoso sexual, mediante hojas de papel escribieron nombres, la descripción y la anécdota del acoso para exhibirlos incluso frente a los propios agresores en lo que algunas llamaron el "muro de la vergüenza" o los "tendederos" colocados en espacios públicos. Este ejercicio se extendió en el país en instituciones públicas y privadas. Es un movimiento con cierta semejanza al \#MeToo, pero totalmente visible, utilizado por estudiantes mujeres como vía para exhibir anónimamente a sus acosadores. Las alumnas de la Universidad Autónoma de Chiapas continuaron con este ejercicio y en varias Facultades y Escuelas aparecieron los tendederos, donde básicamente colgaron de un hilo hojas de papel con una denuncia escrita. El impacto fue el escándalo de realizar señalamientos con nombres de hombres y sus prácticas de acoso que, aunque ya se sabían, en algunos casos fue sorpresa.

Este acto colectivo ha sido una especie de tempero para visualizar el acoso sexual y evidenciar la inaparente violencia de género en cuya intención subyace la idea de promover el cambio de la percepción al toque sensible ante connotaciones integradas a la memoria visual de los perceptores, permitiendo generar miradas que posibiliten ver lo que a simple vista no se puede, instalándose así en la memoria colectiva y por ende se despierte la conciencia.

Esta situación inesperada en el contexto universitario es la manifestación de un síntoma de un conflicto latente -también llamadas por Monereo (2010) como "incidentes críticos"- del "presente viviente" (Richir; 2016) que no se pueden reducir a meras descripciones culturales determinadas por el rompimiento casual de "normas" o valores ideológicos acentuadas de manera paradigmática Es una invitación a apropiarse de un sustrato fenomenológico que posibilite considerar el hecho y el contenido como una extensión de la conciencia de quienes viven cotidianamente la violencia y el acoso. Cada mensaje, es la síntesis de un discurso y la transcripción de una experiencia que amerita exhibirla, en tal sentido Fuster Guillen (2019) afirma que cada “escrito" contiene y expone un sentido latente de violencia que invita a apreciarse como fenómeno indiscutible; que en tanto se conciba como una representación de lo cotidiano da principio al proceso de conocimiento.

El propósito central de este artículo va en ese sentido: Interpretar al tendero como un paisaje lingüístico y semiótico, en la construcción visualizadora de la violencia y acoso sexual en la universidad; entender la esencia del modo de percibir los significados en las construcciones sígnicas en alumnas viviendo una realidad soterrada y cotidiana.

\section{METODOLOGÍA}

El insumo principal, es el tendedero elaborado colectivamente por alumnas en una Facultad de la Universidad Autónoma de Chiapas, ubicada en la ciudad Tapachula, en la frontera sur de México; y específicamente las hojas de papel donde escribieron frases denunciantes del acoso sexual de profesores y profesoras, las colgaron en un hilo para exhibirlos. Se decidió considerar el escenario de los "escritos" bajo el enfoque denominado paisaje lingüístico (PL). Para Martín, Prego, Zas y Taboada (2018) esta noción involucra "el modo en que la lengua se materializa en el espacio público, a través 
de (...) huellas lingüísticas que cumplen funciones tanto informativas como simbólicas en un territorio determinado" (p. 8).

El abordaje del tendedero como un paisaje lingüístico como "una representación que da principio al proceso de conocimiento" alude a la metodología que fue considerar la reflexión resultado de la aplicación de las operaciones de representar e interpretar (facultad de semiósis); del paisaje lingüístico percibido como una construcción sígnica, o bien un texto semiótico. (Sanders; 1992, citado por Flores y Paulin 2019)

En tal sentido, el análisis se abrió en dos líneas: uno, reconocer el acto colectivo de hacer un tendedero, (representación) como un espacio construido para visualizar, descotidianizar y exhibir el acoso sexual y, en un segundo dilucidar el discurso (interpretar), realizando una clasificación de las frases (escritos en los papelitos) que exhiben el sentido de la violencia y el acoso de género.

\section{EL TENDEDERO UN PAISAJE LINGÜÍSTICO QUE DESCOTIDIANIZA EL ACOSO SEXUAL}

El tendedero en sí constituye un paisaje que visualiza los prejuicios, estereotipos e insultos del poder masculino, en tanto que son una evidencia de las representaciones de las mujeres alumnas. Según Jung: “...una palabra o una imagen es simbólica cuando representa algo más que su significado inmediato y obvio. Tiene un aspecto 'inconsciente' más amplio que nunca está definido con precisión o completamente explicado. Ni se puede esperar definirlo o explicarlo. Cuando la mente explora el símbolo, se ve llevada a ideas que yacen más allá del alcance de la razón.” (2002) (citado por Balandray Tenorio; 2011). En tal orden, los "escritos" son la apropiación del espacio y la resistencia a través de señales. Es lingüístico porque existe una "construcción semiótica que visualiza tensiones, conflictos, resistencias y luchas" (Martín, et.al. 2018), "configuran un paisaje semiótico" (Dackow, 2020) que alienta al diálogo entre sí y con otros discursos, porque invita a leerlos para crear conciencia. Este ejercicio colectivo de visualización, -como apunta Cynthia Dackow- irrumpe, desordena, sorprende e incluso usurpa el espacio público con un efecto disruptor. Constituye una práctica y un ejercicio social inusual frente a la violencia de género; demuestra la capacidad colectiva de exhibición en una subjetivación de lo subyacente, lo inaparente de las practicas soterradas de violencia. Constituye nuevas formas que comunidades y sus escenarios diseñan para plantear las contradicciones en una realidad aparentemente normal.

El tendedero visualiza, pone en escena, despoja de la trivialidad y la asume como exhibición tribal del espacio. Este esfuerzo colectivo de visualizar es la intención de demostrar lo que la cotidianidad esconde; se despoja a la violencia y emerge la posibilidad de concientizarse en la percepción de las que lo padecen, que, aunque se sabe, pero no se debe y puede señalarse. Mínguez, Romero y Pedreño (2016) afirman que desde la antropología de Emanuel Levinas, esta descotidianización es la resistencia a ser objeto del "yo pienso" a quedar sometido a su poder; resistencia a "el yo establece una relación de desigualdad respecto al otro": "el yo es rehén del otro". En ese sentido la puesta del tendedero es la insistencia y resistencia en que frente a cualquier intento de que el otro pueda quedar atrapado por la razón o tematizado por una imagen-idea, el rostro del otro queda atrapado en la descripción del papelito. Para Lins Ribeiro (1989) “descotidianizar 
es romper rutinas cotidianas", "es una búsqueda de solucionar la tensión, para revelar, a través de una experiencia totalizante, los elementos constitutivos de la realidad social" (p. 67). En palabras más simples es exigir que la violencia, aunque sea parte de una habituación no puede ni debe seguir siendo así.

El acuerdo colectivo de su puesta es la subjetivación de las conciencias de una práctica moralista reprobable; llevadas a un escenario-tendedero, es en sí una transcripción de la violencia soterrada puesta y escenificada a partir de papelitos que cuelgan de un hilo, estas hojitas de papel es la presencia no física; es la actuación en representación anónima de sus denunciantes, siguiendo el guion que cuenta el enojo, la indignación y el estupor de una cotidianidad agonal.

\section{LA TRANSCRIPCIÓN DE LA EXPERIENCIA DEL ACOSO}

Teniendo como marco teórico el PL, los "escritos" constituyen expresiones semióticas de las experiencias sintetizada en unidades mínimas de significado (Gallegos, 2018). Para su análisis se agruparon en torno a significantes encontrados de acoso: las proposiciones del acoso, el acoso en la frase, el acoso al vestido, la mirada del acoso y, el reclamo por "las otras" y la falta de eticidad. Teóricamente, estos significantes tienen una clara referencia con lo que Cristina Cuenca (2015), denomina el "modelo sociocultural que parte de la idea de que el acoso sexual refleja el desigual reparto de poder práctico y simbólico de hombres y mujeres-, desde y un punto de vista cultural legitimado y de las diferencias de poder entre hombres y mujeres..." (MacKinnon, 1979, citado por Cuenca; 2015, p. 37). Esta autora explica que "Estas sitúan los orígenes del acoso sexual en la sociedad patriarcal"; en tanto que "se percibe como una consecuencia del proceso de socialización de sexo y es un mecanismo por el que los hombres afianzan el poder y el dominio sobre las mujeres", bajo esta noción, "el sexo es un indicador claro que muestra las posibilidades de acoso". En el tratamiento clasificatorio del acoso, se encontraron claramente un listado de comportamientos que las alumnas consideran susceptibles de ser considerados "acoso sexual", asumiéndose así enunciadoras en la manifestación de su saber sobre el acoso sexual.

\section{Tabla 1}

Tabla de clasificación de los tipos de acoso expresados en el Tendedero

\begin{tabular}{|c|c|}
\hline Tipos de acoso & Contenidos en los papelitos \\
\hline $\begin{array}{l}\text { Las proposiciones } \\
\text { del acoso }\end{array}$ & $\begin{array}{l}\text { Si eres virgen yo te puedo ayudar a quitarte la virginidad solo con la puntita. } \\
\text { El profe de ingles siempre me hace comentarios morbosos Te ayudo con tu tesis } \\
\text { a cambio de sexo. } \\
\text {--- egresado me mandaba mensajes en fb para decirme en que lugares me veía y } \\
\text { esperaba la misma combi para seguirme } \\
\text {--- en el extra me dijo que es más fácil acostarme con el que pasar su examen. } \\
\text {--- le pedí eliminar mis msj y número y sigue buscándome a pesar de que no quiero. } \\
\text {--- me ofreció dinero a cambio de sexo. } \\
\text { Egresado: --- me amenazó y compartió fotos intimas con sus amigos. } \\
\text {--- Creo rumores sobre mi persona hace tiempo dijo que era PUTA. }\end{array}$ \\
\hline $\begin{array}{l}\text { Reclamo por las } \\
\text { "otras" }\end{array}$ & $\begin{array}{l}\text { El profesor --- mantiene relaciones con sus alumnas. } \\
\text { Profesor --- acosa a las alumnas. }\end{array}$ \\
\hline
\end{tabular}


Doctor --- acosa a mi amiga personalmente diciéndole que no había dormido esa noche por ver sus fotos.

El profe --- me dijo que si le hacia el favor con una amiga me iba poner 10.

Dr.--- le habla a sus alumnas con la intención de tener sexo.

Doctor --- dijo a mi compañera que no durmió toda la noche por estar viendo las fotos de mi compañera.

--- de --- semestre solo busca a chicas de nuevo ingreso para tener relaciones

--- quiso abusar de mi amiga en estado de ebriedad.

El profesor --- acosa a las alumnas.

Mtro.--- deja de acosar a mis amigas en facebook no es no

El --- acosa a mis compañeras.

--- Igual me insulto diciendo que me saliera de la carrera.

Profesor ---- hace comentarios misóginos hacia las compañeras maestras.

--- dice que tiene el don de adivinar que ropa interior tengo llevo puesta.

El --- me dijo: para que se ponen así, si está durmiendo el niño (Refiriéndose a su pene mientras se rie).

El acoso en la frase

Doctor --- discrimina el trabajo de las maestras, solo por ser mujeres.

Me he sentido acosada como maestra, cuando me silban en los pasillos los alumnos y alumnas.

El maestro --- manda msj sobre el aspecto físico por privado en whats.

--- hace bromas sexuales a alumnas.

El profesor --- hace comentarios machistas.

Mtro. --- estamos cansadas de escuchar sus chistes misóginos y sexistas.

La doctora --- critica la vestimenta de las alumnas.

Dra. --- critica la vestimenta de las alumnas ¡Si quiero llevar shorts a usted que le importa!

El acoso al vestido Dra. --- mi vestimenta no provoca que me acosan, la falta de educación si (no sea de mente cerrada).

La --- me acosaba por mi vestimenta y me amenazo de correrme si venia de short. Dra. --- deje de criticar la vestimenta de mis compañeras.

Mtro.--- morbosea a alumnas enfrente a ella les mira el trasero y se le ha hecho saber a la maestra --- (esposa) y dice que no sean chismosas.

--- sus miradas y comentarios sexistas nos provoca incomodidad. No es sexy es repugnante

--- intento tomarme fotos cuando usaba vestido.

Te paras afuera a lado del chiclerito para estar acosando con la mirada a las estudiantes (---).

La mirada del acoso Dr. --- no sea naco y deje de acosar a sus alumnas, viendo sus piernas y diciendo mamacitas.

El --- del --- (gordo, moreno, con bigote) me acosa con la mirada.

--- del cim mira niñas morbosamente y les tira piropos.

¿Qué no vez? Ella usa falta y tu no (morboso el prof. ----).

Profe --- solo me queda viendo las piernas.

Mtro: --- no mires mis piernas mi falda no es provocativa

--- de --- en un congreso me presiono para ir a su habitación con el pretexto de

Violencia de que íbamos a estar solos y que tenía una botella.

coacción.

--- quisiste obligar a mi amiga a tener sexo en sancris contigo estando ebria.

--- ¿Por qué usaste listón naranja el 25? solo te la pasas burlándote y haciendo comentarios machistas y misóginos. --- dice al grupo los problemas que cuenta en

Falta de eticidad el CAPP

Dra. --- no se puede tener a alguien que dice todo como encargada del capp 
La profesora --- falta al respeto a las estudiantes.

La profesora --- ha hecho comentarios que dañan la persona de profesoras y alumnas.

Fuente: elaboración personal

Los más de cincuenta mensajes, indican que la violencia no es lineal: las hay de maestros a alumnas: Dr. --- no sea naco y deje de acosar a sus alumnas, viendo sus piernas y diciendo mamacitas; de alumnos a maestras: Me he sentido acosada como maestra, cuando me silban en los pasillos los alumnos y alumnas; de maestros a maestras: Doctor --- discrimina el trabajo de las maestras, solo por ser mujeres; de maestras a alumnas: La profesora --- ha hecho comentarios que dañan la persona de profesoras y alumna; de directivos a alumnos: La --- me acosaba por mi vestimenta y me amenazo de correrme si venia de short; y de alumnos a alumnas: --- me ofreció dinero a cambio de sexo ; incluso externos, egresados: --- egresado me mandaba mensajes en fb para decirme en que lugares me veía y esperaba la misma combi para seguirme; y administrativos: El --- del --(gordo, moreno, con bigote) me acosa con la mirada. Pero, hay quien explica: Las protestas no deberían ser anónimas. Mientras que un debate se abre: No estoy de acuerdo con este tendedero ---, y recibe una replica: Pero si estás de acuerdo con meterte en relaciones ajenas.

Hay proposiciones de acoso sexual: Si eres virgen yo te puedo ayudar..., ....me hace comentarios morbosos y, ... en el extra me dijo que es más fácil acostarme con el que pasar su examen, estos exhiben, que más que obtener un favor sexual, la muestra de un poder narcisista al considerar a la mujer como un objeto (sexual); alude a lo que para Marie-France, (1999) se asume: que una mujer está a su disposición, y por lo mismo debe aceptar e incluso sentirse halagada por elegirla; en tanto que no concibe que la mujer codiciada pueda negarse, de lo contrario debe sufrir humillaciones y agresiones.

Por otro lado: Te ayudo con tu tesis a cambio de sexo (---), es la seducción al sexo como quid pro quo (el intercambio de una cosa por otra) que lo remite como elemento de negociación y se establece un dominio que da la impresión de que ambos son beneficiados, sin embargo, es una invitación a una reduccionismo ético y moral de verse como objeto al utilizar al otro (a).

En alusión Pernas (2000) dice "El acoso sexual no es sólo usar el poder para conseguir sexo, sino también usar el sexo para mantener el poder". En esta relación de sexo y poder, el problema no es el sexo ni el poder, sino el abuso del poder como apropiación del otro a través del sometimiento. Es la ofensa del hecho, porque aparte que reduce al cuerpo y toda su sensibilidad, también transforma así en un acto "sagrado" en mercancía u objeto de uso. Hernando Gonzalo (2007) afirma, que quien sostiene su erotismo a través del goce del abuso de poder, transgrede el sentido esencial de la sexualidad, pervierte su destino, deforma su esencia. Marie-France añade que "Apoderarse del cuerpo de otro (su cuerpo físico, emocional y erótico) implica un nivel de violación de la intimidad y del derecho humano que nunca podrá ser explicado a través de ningún concepto de placer por más expandido que pretendamos al erotismo" (1999). La autora explica que el acoso entendido como la invitación a los favores sexuales, es la invitación a depositar el placer en el daño hacia el otro como sometimiento, es una práctica de un narcisismo que impide vivir el placer sin profanarlo apoyado en el "poder" adjudicado. 
Las agresiones son sutiles: ... dice que tiene el don de adivinar que ropa interior tengo llevo puesta. El --- me dijo: para que se ponen así, si está durmiendo el niño (Refiriéndose a su pene mientras se rie); frases que aparentemente no dejan un rastro tangible y que tienden a interpretarse como simples aspectos de una situación de picardía o albur mexicano cuando en realidad, constituyen un intento violento de destrucción moral.

Hay acoso de la mirada en ciertas partes del cuerpo: Profe --- solo me queda viendo las piernas; en el entrecruzamiento de miradas: ¿Qué no vez? Ella usa falta y tu no (morboso el prof. ----); es una afirmación de "yo he visto que la miras y no me gusta como la miras".

La mirada frente a la mirada como dominación: Dra. --- critica la vestimenta de las alumnas; Si quiero llevar shorts a usted que le importa! es un reclamo a la intromisión de la mirada; cuya exigencia de cubrir el cuerpo es sencillamente hostigamiento, humillación, culpabilización, vigilancia constante e insultos, que dan cuenta de una persecución y de ridiculización; son atentados a la autonomía y autodeterminación de la mujer, además que refuerza la ideología de la culpabilización de la mujer, a través de la "naturaleza provocadora” como causa que explica y justifica incluso la violación (Hernández, 2007). Bajo esta representación social está la noción de que la mujer seduce y provoca al hombre a través de conductas, como el modo de vestir que lleva a justificar la acción acosadora y abusadora del varón como respuesta a la provocación. Marie-France, argumenta que los hostigadores sexuales "tienen en común un ideal de rol masculino dominante y unas actitudes negativas con las mujeres y el feminismo-, y se han identificado diferentes categorías de acoso sexual: - el acoso de género, que consiste en tratar a una mujer de un modo distinto porque es una mujer, con observaciones o comportamientos sexistas: el comportamiento seductor, el chantaje sexual y la atención sexual no deseada, la imposición sexual y el asalto sexual" (1999). El imaginario de la frase "ella se lo buscó", es clara referencia a que las actitudes y conductas de las mujeres son interpretadas como un llamado al hombre por lo cual ellas pasan a ser participantes activas de la situación, poniendo en evidencia algo de su deseo. Ana María Fernández (1993) apunta que "suele pensarse la existencia de algo inherente al ser femenino por lo cual la mujer desea este "reconocimiento" del hombre, el cual con su mirada la define y alimenta su narcisismo", lo que sostiene la creencia de que las mujeres disfrutan con estas situaciones y por eso buscan generarlas.

El acoso es una insistencia: --- le pedí eliminar mis msjy número y sigue buscándome a pesar de que no quiero. El acoso por un servicio sexual. --- me ofreció dinero a cambio de sexo; el acoso provocando escozor: --- sus miradas y comentarios sexistas nos provoca incomodidad. No es sexy es repugnante. Son reacciones que deben ser analizados desde las razones del desagrado de la insinuación sexual porque precisamente esto es acoso: insinuaciones cargadas de intenciones, expresadas inoportunamente que indudablemente genera desagrado, aun cuando se oculta, se ahoga, y no llega a ser verbal, el acoso transpira a través de las miradas insistentes, comentarios admirativos, incluso un albur; esas insinuaciones reticentes y silenciosas son las expresiones de un acosador sexual.

Martha Lamas (2019) explica que esto no es tan sencillo explicar esta urdidumbre cultural porque expresar interés sexual sin que se solicite se califica como acoso, pero no lo es cuando para lograr una atención sexual que sí se desea hay que recibir y dar una 
buena cantidad de atención sexual no deseada. Lamas (2019) revela que, desde una lectura antropológica, por mandato cultural los hombres "conquistan" a las mujeres, manifestando interés y requerimiento sexual; el "abordaje" se hace a través de "proposiciones" que es una iniciativa que engloba al cortejo masculino, porque en nuestra cultura la iniciativa sexual les corresponde. La autora, "afirma que hay códigos culturales que aconsejan que con las mujeres hay que insistir, pues las "decentes" siempre deben decir que no... al principio. Aunque a veces el acoso radica en el empleo nocivo del lenguaje, simplemente comunican exactamente lo opuesto".

La burla y el sarcasmo también están presente, en el denominado lenguaje misógino: Doctor --- discrimina el trabajo de las maestras, solo por ser mujeres. El maestro --- manda msj sobre el aspecto físico por privado en whats. -- hace bromas sexuales a alumnas. El profesor --- bace comentarios machistas. Mtro. --- estamos cansadas de escuchar sus chistes misóginos y sexistas. En estas alusiones existen elementos de un juego verbal, que expresa placer al nombrarlas en la descripción de asuntos propios de las mujeres, pero con exageración, siempre en detrimento en su condición de mujer.

La transcripción del acoso en "las hojitas", permite entender las experiencias subjetivadas, la violencia se establece de un modo insidioso tan difícil de identificarla. Defenderse de manera individual es difícil, pero de manera colectiva resultar necesaria siempre es posible, en el tendedero se puede decir que la violencia atenta contra una mujer; atenta contra la dignidad de todas, estas prácticas visualizadoras dan cuenta a tiempo que los límites estaban siendo rebasados, y no han sabido hacerse respetar.

\section{Reclamo por la no eticidad.}

La violencia exhibida no solo es de acoso, también es un reclamo para no cumplir con ese marco de acción depositadas moral y socialmente con valores asignados a la función y principios prescritos de ser maestro. En tal sentido hay un reclamo que expresan indignación por la difusión de lo que es intimo o privado, fotos, mensajes y el hecho de "hablar" del cuerpo. Reclamo por simular "¿Por qué usaste listón naranja...?". También se reclama por: "yo sé", de lo que la "otra no puede decir". Es un reclamo a las indiscreciones. --- dice al grupo los problemas que cuenta en el CAPP. Dra. --- no se puede tener a alguien que dice todo como encargada del capp. Para el colectivo, la violencia radica en la no eticidad para contar lo que se sabe, es un reclamo de violentar la intimidad al exhibir lo que se dijo en confesión.

Los acosadores no solo son hombres y maestros, son mujeres maestras, directivas, compañeros: tienen nombre y apelativo. La profesora --- falta al respeto a las estudiantes. La profesora --- ha hecho comentarios que dañan la persona de profesoras y alumnas. En referencia clara al hecho, no a la condición de género.

Este reclamo es por lo que Carlos Ruiz asume como "abandono de la eticidad, por soslayar el sentido normativo o de una suerte de código normativo, de un código operante y sostenido por el modo de vida efectivo de esta comunidad". Recordemos que la eticidad es la mezcla de valores éticos y morales propios de una identidad asumida, el maestro al querer y ser maestro asumió un código operante y en tanto que, la eticidad vaya más allá del simplemente ser, implica comportarse como tal; bajo esa sentencia un maestro -desde 
la eticidad- no es tal porque tenga herramientas didácticas y pedagógicas; sino porque se asume como tal y asumir es demostrarlo.

El reclamo no se puede tener a alguien... es un señalamiento de no tener eticidad frente a situaciones socio-morales donde se deben asumir conductas ética-morales. Es un señalamiento frente a una eticidad deficitaria que reproducen estructuras sociales injustas.

Marie-France, H. (1999) afirma que esos pequeños actos de violencia son tan cotidianos que parecen normales; empiezan con una sencilla falta de respeto o con una manipulación que sólo son insoportables si afectan directamente; luego, si el grupo social en el que aparecen no reacciona, estos actos se transforman progresivamente en verdaderas conductas perversas que tienen graves consecuencias que al no tener la seguridad de que serán comprendidas.

\section{CONCLUSIONES}

Si la violencia es parte de la cotidianidad en la universidad, entonces el tendedero, como practica de visualización es una descotidianización, es una ruptura con el flujo de la regularidad cotidiana de la violencia; pues evidencia lo que se sabe pero que no se admite: los espacios universitarios, las aulas, las clases, las materias y las interacciones entre alumnos y maestros, están cargadas de violencia. Alumnas y maestros viven y conviven en la violencia: son sujetos violentos y objetos de violencia. La violencia de género y el acoso sexual son parte de una urdidumbre simbólica, exhibida en un paisaje lingüístico que expresa en las frases, en las miradas y en la atmosfera que encuentra en la universidad un terreno fértil, escasamente visibilizada y que además de vigente, con alta prevalencia, manifestada verbal y gestual; se sostiene por la no denuncia asida a relaciones laborales de miedo y desconfianza además de la desprotección y desatención institucional.

En tal sentido, en la universidad se aprende a vivir en la violencia, a ser violento, a ser violentado y perpetuar la violencia, bajo representaciones del orden patriarcal que se discurren en la cotidianidad asumiendo y perpetuando tal concepción. María Torres Mora, citando a Pierre Bourdieu afirma que “(...) la educación es reproducción, es decir, una actividad que colabora para conservar el estado de cosas en una sociedad y al hacer esto ayuda a que se reproduzca, a que se mantenga y perpetúe el orden social" (2010;32) en consecuencia "la educación inculca el arbitrio cultural de un sector dominante", lo que viene a ser una forma de violencia: la simbólica. "Es simbólica porque los educandos desconocen que se les está inculcando como algo "natural", una lógica que favorece el orden establecido; por un lado, impone arbitrarios culturales y, por otro, descalifica y deslegitima saberes, estilos de vida, intereses grupales e individuales que no correspondan a los modelos dominantes" (Torres, 2010; p. 28).

Estas prácticas visualizadoras del tendedero permiten que se vaya construyendo una delimitación de que actos deben ser considerados como "acoso" y asumidos como tal por las comunidades universitarias. Sin embargo, deben ser objetos de constante reflexión para no caer en el enardecimiento estudiantil y en presión política para atentar contra derechos de terceros. El problema del acoso esta precisamente en la hipersusceptibilidad de los actos con ciertos prejuicios que originan señalamientos e injusticias; el discurso que califica lo funcional como dispositivo que, al estar centrado obsesivamente en la 
"depravada" sexualidad masculina, puede olvidar todo lo que se sabe ya acerca de las complejidades y ambigüedades presentes en las relaciones humanas (Lamas, 2019), además que facilita pervertir el sentido del tendedero. Al obviar esas precisiones se corre el riesgo de desplazar problemas de lo social al campo de la sexualidad. Hay un peligro latente no sólo analítica sino también política, puesto que existe la posibilidad de que se reconozca que la sexualidad es más determinante que otras conductas sociales y culturales como la violencia y el abuso del poder.

En suma, el tendedero, evoca temas que pueden ser abordados en extenso desde las categorías de poder, conflicto, subversión o resistencia, pero también de manera promisoria concibe a la escuela como un espacio de producción de discursos, prácticas liberadoras y emancipadoras. Por el momento, la riqueza empírica de los "escritos" debe ser una manera de sensibilizarnos para promover una sociedad más justa y equitativa.

\section{REFERENCIAS BIBLIOGRÁFICAS}

Arias, Adriana, (2011) Sexo y poder: una relación compleja, en: El clarin.com. periódico digital, 19/05/2011. https://www.clarin.com/psicologia/sexo-poder-usoabusoadriana-arias_0_SkrM8ecvXl.html

Balandra, A. C. Á., \& Tenorio, V. Á. (2011) La Eticidad en la Formación de Investigadores Educativos. REICE. Revista Iberoamericana sobre Calidad, Eficacia y Cambio en Educación, 9(2).

Castro, R. y Vázquez García, V. (2008) La Universidad como espacio de reproducción de la violencia de género. Estudio de caso en la Universidad Autónoma Chapingo, México. Estudios sociológicos XXVI: 78, 2008.

Cuenca Piqueras, Cristina. (2015). Factores precipitantes del acoso sexual laboral en España. Revista mexicana de sociología, 77(4), 525-554., http://www.scielo.org.mx/scielo.php?script=sci_arttext\&pid=S0188-2503201500 0400525\&lng $=\mathrm{es} \& \operatorname{tn} \mathrm{ln}=\mathrm{es}$.

Dackow, Cynthia (2020). Paisajes Semióticos Disidentes. La retórica de los feminismos del 8M y 9M. https://www.aacademica.org/cynthia.dackow/4.pdf

D’Amore, B. (2006). Objetos, significados, representaciones semióticas y sentido. RELIME. Revista latinoamericana de investigación en matemática educativa, 9(1), 177-196.

Depraz, Natalie. (2001). The Husserlian theory os intersubjectivity as alterology: Emergent theories and wisdom traditions in the light of genetic phenomenology. En Thompson, Evan (ed.), Between Ourselves. Second-person issues in the study of consciousness (169-178).

Fernández, A. M. (1993). La mujer de la ilusión: pactos y contratos entre hombres y mujeres (Vol. 24). Buenos Aires: Paidós.

Flores Mercado, B. G., \& Paulin Perez, G. (2019). Sociosemiótica y cultura: principios de semiótica y modelos de análisis. UNAM, Instituto de Investigaciones Sociales. 
Fuster Guillen, D.E. (2019) Investigación cualitativa: Método fenomenológico hermenéutico: Propósitos y Representaciones Ene.- Abr. 2019, Vol. 7, N 1: pp. $201-229$

Gallegos Krause, Eduardo (2018). De la semiótica textual a la semiótica del discurso: (dis) tensiones entre tradición y novedad. deSignis, 29 0, 229-236. https://www. redalyc.org/articulo.oa?id $=606065855022$

Gonzalo, A. H. (2007). Sexo, Género y Poder. Breve reflexión sobre algunos conceptos manejados en la Arqueología del Género. Complutum, 18, 167.

Hiner, H. (2009). Voces soterradas, violencias ignoradas: Discurso, violencia política y género en los Informes Rettig y Valech. Latin American Research Review 44 (3), 50-74. doi: 10.1353/lar.0.0082.

Krause, E. G. (2018). De la semiótica textual a la semiótica del discurso:(dis) tensiones entre tradición y novedad. DeSignis, 29, 229-236. https://www.redalyc.org/ journal/6060/606065855022/html/

Lamas, M. (2019). El acoso y el\# metoo. Revista de la Universidad de México, (2), 53-59.

Ribeiro, G. L. (1989). Descotidianizar. Extrañamiento y conciencia práctica. Un ensayo sobre la perspectiva antropológica. Cuadernos de antropología social, (3).

María Teresa Torres Mora. (2010) El problema de la violencia entre universitarios abordado desde el enfoque de la Investigación- Participación-Acción. En: Investigación Universitaria Multidisciplinaria 9 (9), 27-36

Marie-France, H. (1999). El acoso moral El maltrato psicológico en la vida cotidiana. Ediciones Paidós Ibérica, S.A.,Barcelona.

Martín R. L., Prego, G., Zas L., Taboda M.B. (2018) Observatorio del discurso. https:// www.edisoportal.org/attachments/article/796/ObservatoriodelDiscurso_junio20 18.pdf

Mínguez V. R., Romero S.B.E., \& Pedreño P.M. (2016). La pedagogía del otro: bases antropológicas e implicaciones educativas. 163-183.

Monereo, C. (2010). La formación del profesorado: una pauta para el análisis e intervención a través de incidentes críticos. Revista Iberoamericana de educación, 52(1), 149-178.

Muñoz-Saavedra, J. (2019) Una nueva ola de feminista... más allá de \#Me'Too. Irrupción, legado y desafíos. En P. Rivera-Vargas, J. Muñoz-Saavedra, R. Morales Olivares y S. Butendieck-Hijerra (Ed.). Políticas Públicas para la Equidad vol II.

[Preprint] 2019. Santiago de Chile: Colección Políticas Públicas, Universidad de Santiago de Chile

Pernas, B.; Olza, M.; Naredo, M. J. La dignidad quebrada. Las raíces del acoso sexual en el trabajo. Madrid: Libros de la Catarata, 2000.

Ruiz S., C. (1999). El concepto hegeliano de eticidad y el comunitarismo. https://repositorio.uc.cl/xmlui/bitstream/handle/11534/9965/000375953.pdf 
Tapia H., S.I. (2015) Violencia de género en las universidades o la necesidad de una intervención educativa International. Journal of Developmental and Educational Psychology, 1, (1), 2015, 531-543

Zamudio S., F.J.; Andrade B., M.A.; Arana O., R.I. \& Alvarado S., A A. (2017). Violencia de género sobre estudiantes universitarios(as). Convergencia, 24(75), 133-157.

\section{CITAR COMO:}

Chan Pech, C. (2021). Visualización del acoso en tendederos universitarios; una construcción de paisajes lingüísticos. Puriq, 3(4), 622-634. https://doi.org/10.37073/puriq.3.4.229 